\title{
Adsorption methodology: Synthesis of Nano- structured nitrogen-doped porous carbon adsorbents for perchloroethylene vapor adsorption
}

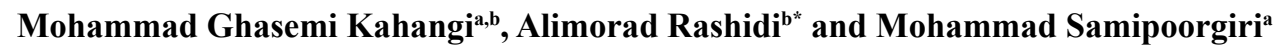 \\ ${ }^{a}$ Chemical Engineering Department, Islamic Azad University, North Tehran Branch, Tehran, Iran \\ ${ }^{b}$ Carbon and Nanotechnology Research Center, Research Institute of Petroleum Industry, Tehran, Iran
}

\section{A R T I C L E I N F O :}

Received 12 Aug 2020

Revised form 5 Oct 2020

Accepted 12 Nov 2020

Available online 30 Dec 2020

\section{Keywords:}

Perchloroethylene,

Adsorption procedure,

Gas chromatography mass spectrometry,

Porous Nano carbon,

Nitrogen-doped adsorbent.

\begin{abstract}
A B S T R A C T
The perchloroethylene (PCE, tetrachloroethylene), as a representative of chlorinated ethylenes and volatile organic compounds (VOCs), can be easily transported and remain in the atmosphere due to its volatility and stability properties. As a result, there is a crucial need to reduce this pollution to the extent permitted by international standards. The concentration of PCE determined with Gas chromatographymass spectrometry analyzer (GC-MS). Activated nanocarbons (ACs) doped with nitrogen functional groups were prepared using the walnut shell as a precursor to evaluate their adsorption capacity for PCE vapors. Several techniques, including scanning electron microscopy (SEM), $\mathrm{N}_{2}$ adsorption-desorption, and the Fourier transforms infrared spectrometry (FTIR), were applied to characterize the physical-chemical properties of the ACs. It is found that the PCE adsorption considerably increased on the nitrogen-doped ACs (KNCWS) due to their structural and surface charge properties. By conducting kinetic study, the pseudo-first-order model matched well with experimental data that could indicate reversible adsorption of the PCE on heteroatom doped ACs. The sips model agreed well with the equilibrium adsorption of PCE on the nitrogen-doped ACs, and the maximum adsorption capacities for PCE reached 166, 285, and 95 $\mathrm{mg} / \mathrm{g}$ for KNCWS-11, KNCWS-21, and KNCWS-31, respectively. Also, the concentration of PCE were online measured based on nitrogen-doped ACs as solid-phase extraction (SPE) by the GC-MS as analytical procedure. Therefore, the nitrogen-doped ACs was good choices for the removal of PCE vapors.
\end{abstract}

\section{Introduction}

Atmospheric air, as an important topic of the environment that ensures the lives on Earth, not only contains natural and vital compounds, but also a set of undesirable and unnatural materials [1].

\footnotetext{
* Corresponding Author: A.M. Rashidi

E-mail addresses: rashidiam@ripi.ir

https://doi.org/10.24200/amecj.v3.i04.125
}

Since the existence of volatile organic compounds (VOCs) in the atmosphere can cause severe health problems. They have attracted a lot of attention and many efforts have been made to removal them in recent years [2]. In addition, numerous VOCs contribute to the degradation of the stratospheric ozone layer, which is part of climate change. VOCs are also considered a precursor to the secondary formation of PM2.5 particulate 
matter [3]. According to various sources, volatile organic compounds in atmospheric air are divided into several main groups of alkanes, alkenes, alkynes, aromatics, volatile organic compounds of chlorine, and volatile organic compounds of sulfur. Chlorine volatile organic compounds are much more environmentally toxic than other VOCs. Specifically, Cl-VOCs are primarily known as stable, biogenic, and biodegradable compounds in the environment. Most of them are toxic, and carcinogenic. Therefore, today we need to research effective techniques for managing currents contaminated with $\mathrm{Cl}$-VOCs that are economically viable [4]. Perchloroethylene (PCE, tetrachloroethylene) as a representative of chlorine ethylenes is a synthetic chlorine hydrocarbon known for its exceptional solubility and low inflammability. PCE is a colorless and sweet-smelling liquid with highly solubility in water that is widely utilized as a solvent in industrial processes like metal degreasing, drying, and drug, pesticides, adhesives, and antifreeze production [5]. Due to the prevalence of leakage and its inappropriate disposal, large amounts of PCE enter the environment in industrial sites [6]. PCE with a vapor pressure of $18.5 \mathrm{~mm}$ $\mathrm{Hg}$ at $25^{\circ} \mathrm{C}$ is predicted to exist only as vapor in the ambient atmosphere [7]. According to OSHA, PCE levels above $100 \mathrm{ppm}$ can cause neurological effects that can damage to the central nervous system in humans [8]. Many methods have been reported to control environmental pollution, such as adsorption, advanced oxidation, electrochemical and photocatalytic method. Because of low cost, simplicity in design, and operation, the adsorption method is widely used among the various methods to remove the $\mathrm{Cl}$-VOCs. Cost-effective, efficient, and sustainable methods for removing VOCs are still a challenge and need more attention in this regard. Zeng et al. (2015) found that activated carbon aganite-nanocomposite ( $\beta$-FeOOH-AC) has outstanding capacity for PCE adsorption. It is shown that PCE removal with an adsorbent dose of $8 \mathrm{~g} / \mathrm{L}$ and an initial concentration of $100 \mathrm{mg} / \mathrm{L}$ can reach $97.83 \%$. Adsorption kinetics indicates that the quasi-second-order model fits well with the experimental data. The Langmuir and Freundlich isotherm models were reasonably fitted to describe the PCE adsorption behavior on $\beta-\mathrm{FeOOH}$ $\mathrm{AC}$ from water. The thermodynamic results of adsorption show that the PCE adsorption process on $\beta-\mathrm{FeOOH}-\mathrm{AC}$ is a spontaneous, endothermic, monolayer, and multilayer adsorption process joint with a physical process that occurs through an ion-exchange surface adsorption mechanism [9]. In the adsorption process, the price of the adsorbent is one of the most important factors in the economics of the process, so most researchers try to build and select a cheap adsorbent in this process [10]. Lignocellulose wastes are a good option for cheap adsorbents, as the existing wastes enter efficient cycles. Activated carbon used to prevent normal landfilling in landfills is recyclable and economically competitive with other methods. In addition, the solid phase extraction as analytical techniques, such as the needle trap extraction, the tube extraction, the sorption trap, solid-phase microextraction (SPME), the thin-film extraction, dynamic SPE, and stir bar SPE were used for determination PCE and VOCs in air by GC-MS.

This method has the dual advantage of reducing waste production and greenhouse gas emissions. Accordingly, In this research, by using the walnut shell as a precursor, a series of urea modified carbons were activated by $\mathrm{KOH}$ and the effect of in situ $\mathrm{N}$-doping on the textural properties as well as PCE adsorption performance were investigated. In addition the proposed procedure based on nitrogendoped ACs coupled to GC-MS analyzer. Moreover, Different analysis (BET, FE-SEM, and FT-IR) were employed to characterize the as-prepared ACs.

\section{Experimental}

\subsection{Apparatus and Reagents}

The gas chromatography (GC) equipped with loop injector was used for etrachloroethylene analysis in air (Agilent GC, 7890A, GC-MS, Netherland). The detector of triple quadrupole (MS) have used in Agilent GC. Due to injection process, the slide of plunger carrier down and tighten the plunger thumb screw until finger-tight. The sampling 
valves introduce a sample into the carrier gas stream and valves were used to inject a sample gas in gas streams. Iranian walnut shell was used as the precursor of nanocarbons. Moreover, $\mathrm{KOH}$, urea, and $\mathrm{HCl}$ with analytical grade were purchased from Merck. The etrachloroethylene (perchloroethylene, PCE) was purchased from Sigma Aldrich ([ $\left.\mathrm{HOCH}_{2} \mathrm{CH}_{2} \mathrm{~N}\left(\mathrm{CH}_{2} \mathrm{COOH}\right)\right]$; CAS Number: 9362-9). Acetone and ethanol prepared from Sigma, Germany.

\subsection{Preparation of $N$-doped nanocarbons}

Walnut shell was respectively crushed, washed, and dried at $100{ }^{\circ} \mathrm{C}$ for 24 hours. The precursor was powdered and sieved into $150 \mu \mathrm{m}$. The carbonization of powdered WS was carried out under nitrogen gas stream by a heating rate of $5{ }^{\circ} \mathrm{C} /$ $\min$ to $600{ }^{\circ} \mathrm{C}$ for about 1 hour (CWS). Then ureaas a source of nitrogen-was mixed with different ratios of carbonized samples $(1: 1,2: 1$, and 3:1) and heated at $400^{\circ} \mathrm{C}$ for 1 hour through a heating rate of $3.33{ }^{\circ} \mathrm{C} / \mathrm{min}$ (NCWS). Next, the nitrogendoped carbon was mixed with $\mathrm{KOH}$ with the ratio of $1: 4$ and activated under $\mathrm{N}_{2}$ using a heating rate of $7.5^{\circ} \mathrm{C}$ per minute up to $900{ }^{\circ} \mathrm{C}$ for 1 hour. The as-prepared samples were washed with $1 \mathrm{M} \mathrm{HCl}$ and hot distilled water until neutral $\mathrm{pH}$ and finally were dried in the vacuum oven at $80^{\circ} \mathrm{C}$ for $5 \mathrm{~h}$. The as-prepared samples were denoted as KNCWS-xy in which xy represents the ratio of urea to CWS.

\subsection{Characterization}

To investigate the specific surface area, pore diameter, and total pore volume, adsorption/ desorption isotherm of nitrogen was performed using a Micromeritics ASAP 2020 Plus device. Each sample was degassed at vacuum at $250{ }^{\circ} \mathrm{C}$ and nitrogen uptake was performed at $196^{\circ} \mathrm{C}$. To provide the morphology of as-prepared ACs, a TESCAN MIRA3 field emission scanning electron microscope (FE-SEM) was used. Reflectance fourier transform infrared (FT-IR) spectra were collected from the absorbance intensities of functional groups of as-prepared ACs by Thermo Nicolet AVATAR 360 within the $4000-400 \mathrm{~cm}^{-1}$ wave number range.

\subsection{Procedure}

Figure 1 shows a schematic of a PCE adsorption laboratory system operating according to the law of thermodynamic equilibrium between the liquid and gas phases. This device is used to absorb PCE in the range of $0-1000 \mathrm{ppm}, 25^{\circ} \mathrm{C}$, and atmospheric pressure.

Continuous $\mathrm{N}_{2}-\mathrm{PCE}$ flow is produced by the flow of $\mathrm{N}_{2}$ through a liquid PCE tank to obtain a certain concentration of PCE in the gas stream by changing the ratio of concentrated and dilute $\mathrm{N}_{2}$ flow. Concentrated $\mathrm{N}_{2}$ flows out of the bubble tank and flows continuously onto the activated carbon sample located in the adsorbent bed. Contaminated $\mathrm{N}_{2}$ passed through the adsorbent and the process

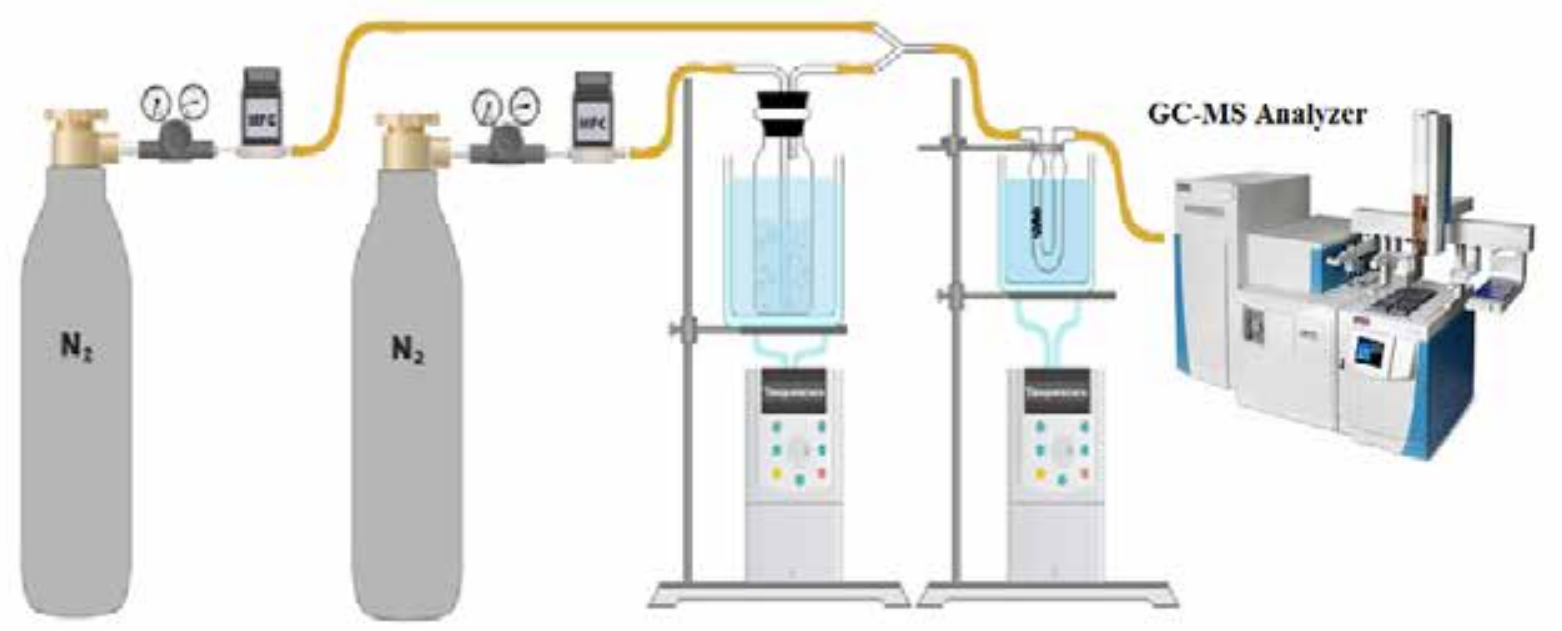

Fig. 1. Schematic of the PCE adsorption laboratory system 
continued until the adsorbent saturated with PCE and the gas flow at the inlet and outlet of the column was analyzed online with GC-MS analyzer. Using the refractive index, the time required for saturation can be achieved [11].

\section{Results and Discussion}

\subsection{FE-SEM}

SEM micrographs of the carbonized walnut shell (Fig. 2a) has a smooth, pore-free surface in which, after nitrogen doping (Fig. 2b), a number of macropores are seen on the sample. Figure 2c shows a spongy structure containing uniform, very fine cavities of micro and nano size, showing that severe morphological changes have occurred during the activation process and that the active nano carbons bear no resemblance to the carbonized sample. To better understand the textural properties of activated nano carbons, the surface was analyzed by physical adsorption of $\mathrm{N}_{2}$.

\subsection{Physical Properties}

$\mathrm{N}_{2}$ adsorption/desorption isotherms were measured to evaluate the textural properties of the samples. The adsorption isotherms of Figure 3a are of type I according to the IUPAC classification, and its desorption is of type H4. According to research, $\mathrm{H} 4$ type hysteresis rings are related to the narrow slit of the specimen [12]. The isotherms have a soft curvature in the relative pressure between 0 and 0.4 owing to the presence of cavities larger than the average adsorbed diameter. On the other hand,
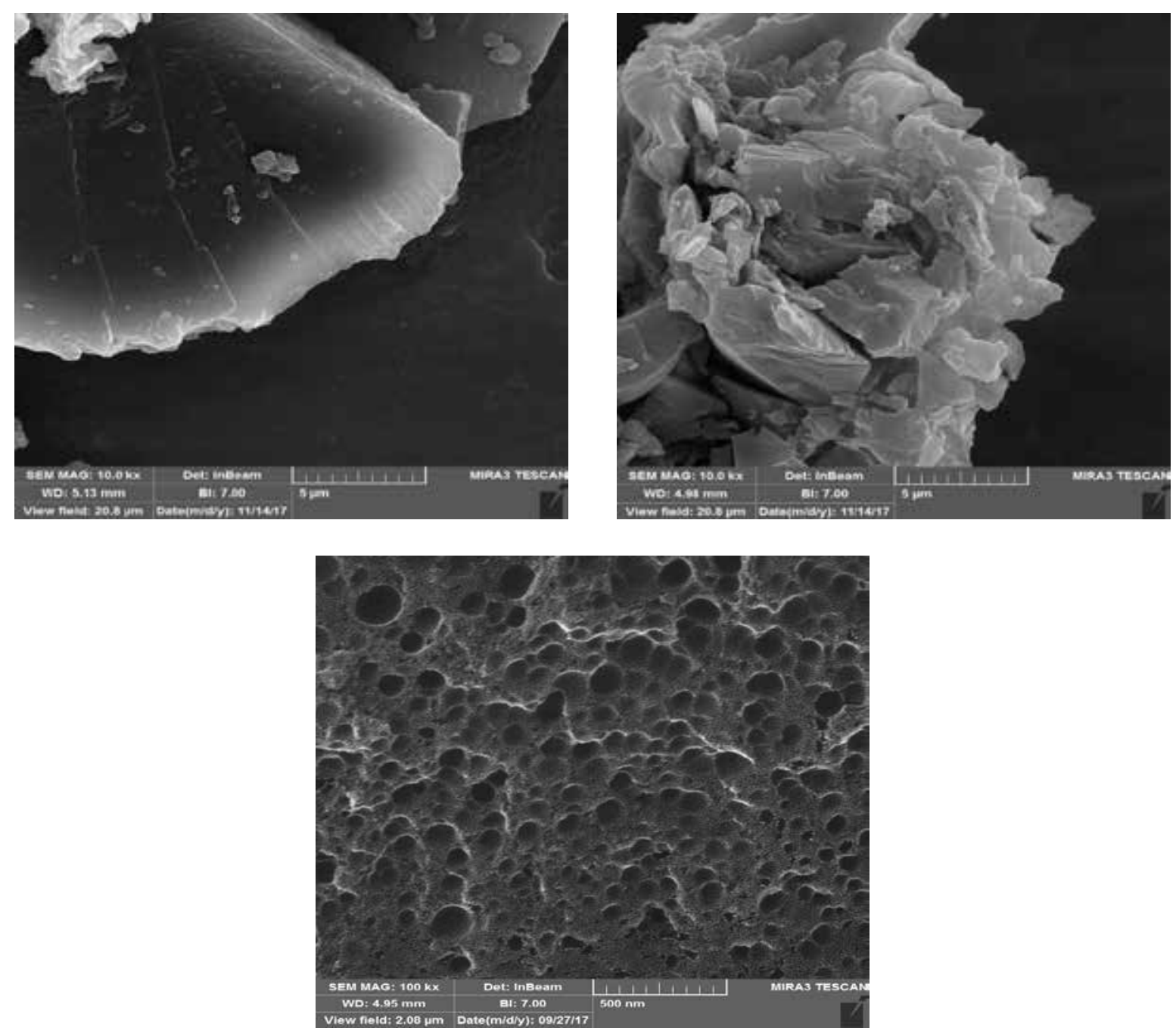

Fig. 2. FE-SEM images of a) CWS, b) NCWS-21, c) KNCWS-21 
more curvature of the isotherm knee at low relative pressure and a slight increase in $\mathrm{N}_{2}$ adsorbed with increasing pressure indicate expansion (enlargement) of the micropore structure. The hysteresis ring exhibited at a relative pressure of about 0.5 indicates the definite presence of mesocavities in the samples $\left(\mathrm{P} / \mathrm{P}_{0}>0.4\right)$ [13]. As can be seen from the wider circle of KNCWS-21 and KNCWS-11 hysteresis, the participation of mesopores is greater. This is in line with our findings, which show a maximum average pore width of $2.48 \mathrm{~nm}$ for KNCWS-21 and $2.41 \mathrm{~nm}$ for
KNCWS-33 [14]. Based on the distribution shown in Figure $3 b$, the cavities mainly contain sizes less than $10 \mathrm{~nm}$, which indicates the presence of both micropores and mesopores. With increasing the urea to carbon ratio from $1: 1$ to $2: 1$, the peak intensity in the pore distribution curves of the samples increased and then decreased to a ratio of $3: 1$. The degradation of the structural parameters of the KNCWS-31 sample compared to KNCWS-21 and occurred due to the destruction of the walls between the cavities or the blockage of the cavities with excessive nitrogen [15] (Table 1).

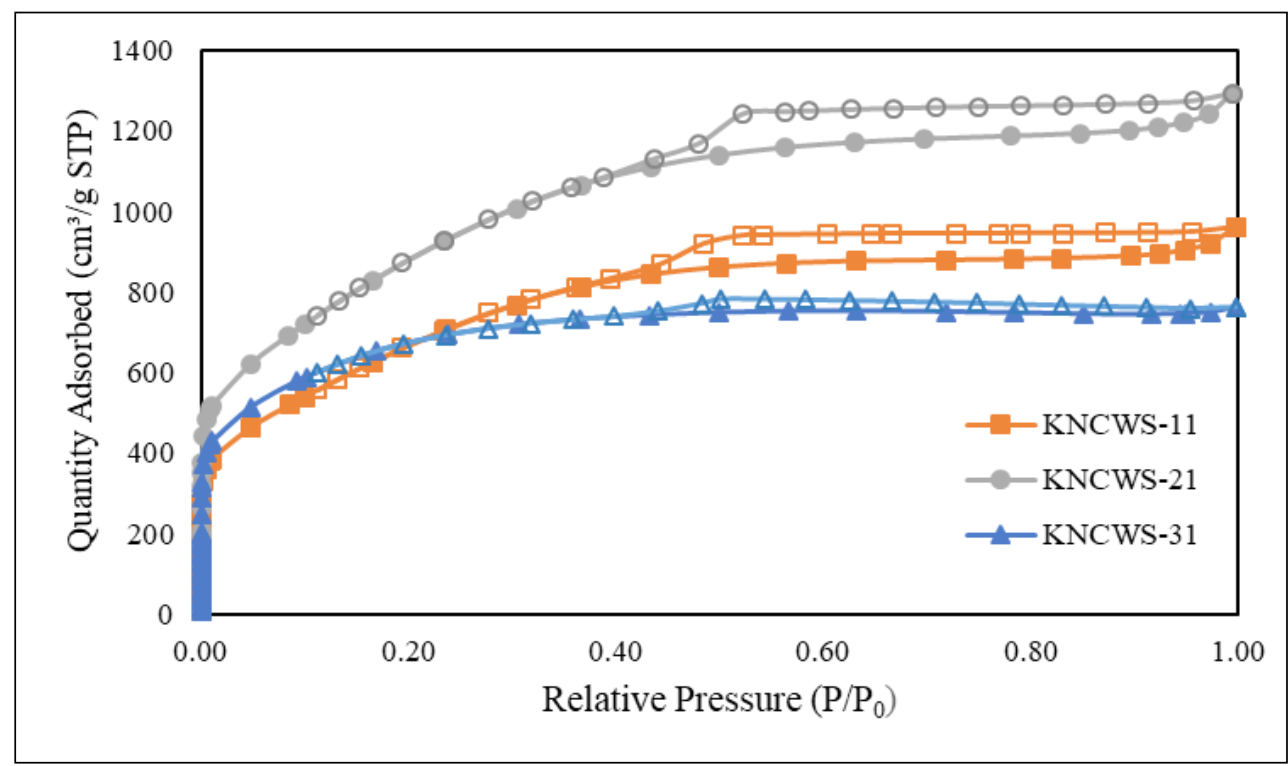

(a)

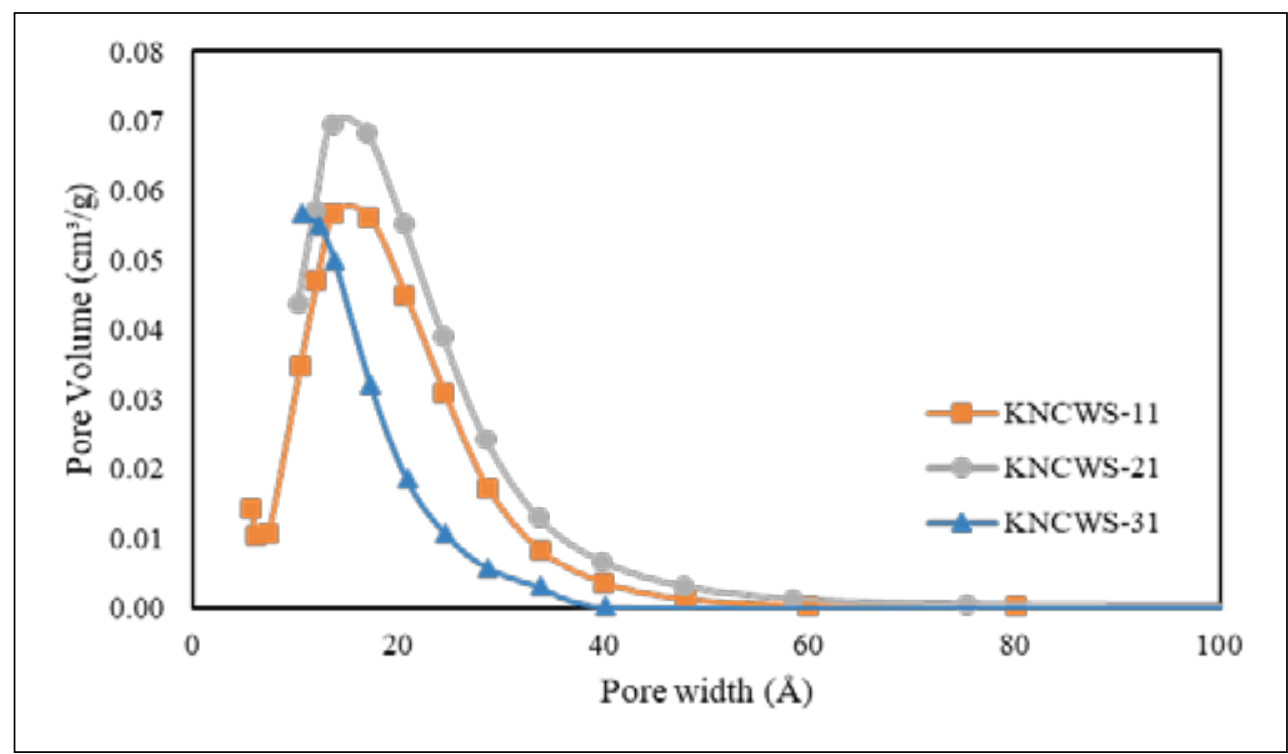

(b)

Fig. 3. $\mathrm{N}_{2}$ adsorption/desorption isotherms(a) and BJH pore size distribution of $\mathrm{KNCWSs}(\mathrm{b})$. 
Table 1. Textural properties of the KNCWSs

\begin{tabular}{ccccc}
\hline Specimens & $\mathbf{S}_{\text {BET }}$ & $\mathbf{D}$ & $\mathbf{V}_{\text {tot }}$ & $\mathbf{V}_{\text {mic }} / \mathbf{V}_{\text {tot }}$ \\
\cline { 2 - 5 } & $\mathbf{( \mathbf { m } ^ { 2 } / \mathbf { g } )}$ & $\mathbf{( n m )}$ & $\mathbf{( \mathbf { c m } ^ { 3 } / \mathbf { g } )}$ & $\mathbf{( \% )}$ \\
\hline KNCWS-11 & 2461 & 2.41 & 1.48 & 8.78 \\
\hline KNCWS-21 & 3225 & 2.48 & 1.99 & 10.05 \\
\hline KNCWS-31 & 2319 & 2.03 & 1.17 & 20.5 \\
\hline
\end{tabular}

\subsection{FT-IR}

The diagrams in Figure 4 are the results of the FT-IR tests (a) CWS, (b) NCWS-21, and (c) KNCWS-21. The samples show almost the same spectrum, however, some of the weak/strong functional groups have disappeared. Nitrogen doping as well as different activation conditions have resulted in changes in the carbon sample spectrum. 3426 3409 $\mathrm{cm}^{-1}$ bandwidth is seen for all samples, indicating tensile vibration of $\mathrm{N}-\mathrm{H}$ groups or tensile vibration of O-H hydroxyl groups of phenol, alcohol, and carboxylic acid [16]. The broadband in the range of $2922 \sim 12851 \mathrm{~cm}^{-1}$ is related to the asymmetric tensile vibration of $\mathrm{CH}_{2}$, which can be attributed to the - $\mathrm{CH}$ bond on the carbon surface. - $\mathrm{CH}$ - bonds may belong to alkyl groups such as methyl, methylene groups, or aldehyde groups [17]. The bandwidth at 1612
$11376 \mathrm{~cm}^{-1}$ is due to amides, pyridine, and $\mathrm{C}=\mathrm{N}$, indicates nitrogen functional groups at temperatures higher than crude carbon [18]. The peak range of $1030 \sim 1099 \mathrm{~cm}^{-1}$ is also attributed to the C-N tensile vibration [19]. Therefore, analysis of FT-IR spectra confirms the presence of $\mathrm{N}$-containing groups in the synthesized samples.

\subsection{Equilibrium adsorption}

Figure 5 shows the performance of both chemical adsorption processes (nitrogen doping) and physical adsorption (increasing surface area and pore volume). Due to the trend of PCE adsorption isotherms, intensification of carbon activation has improved the adsorption process. Also, increasing the adsorption of PCE by adding nitrogen plays an important role for functional groups, because the

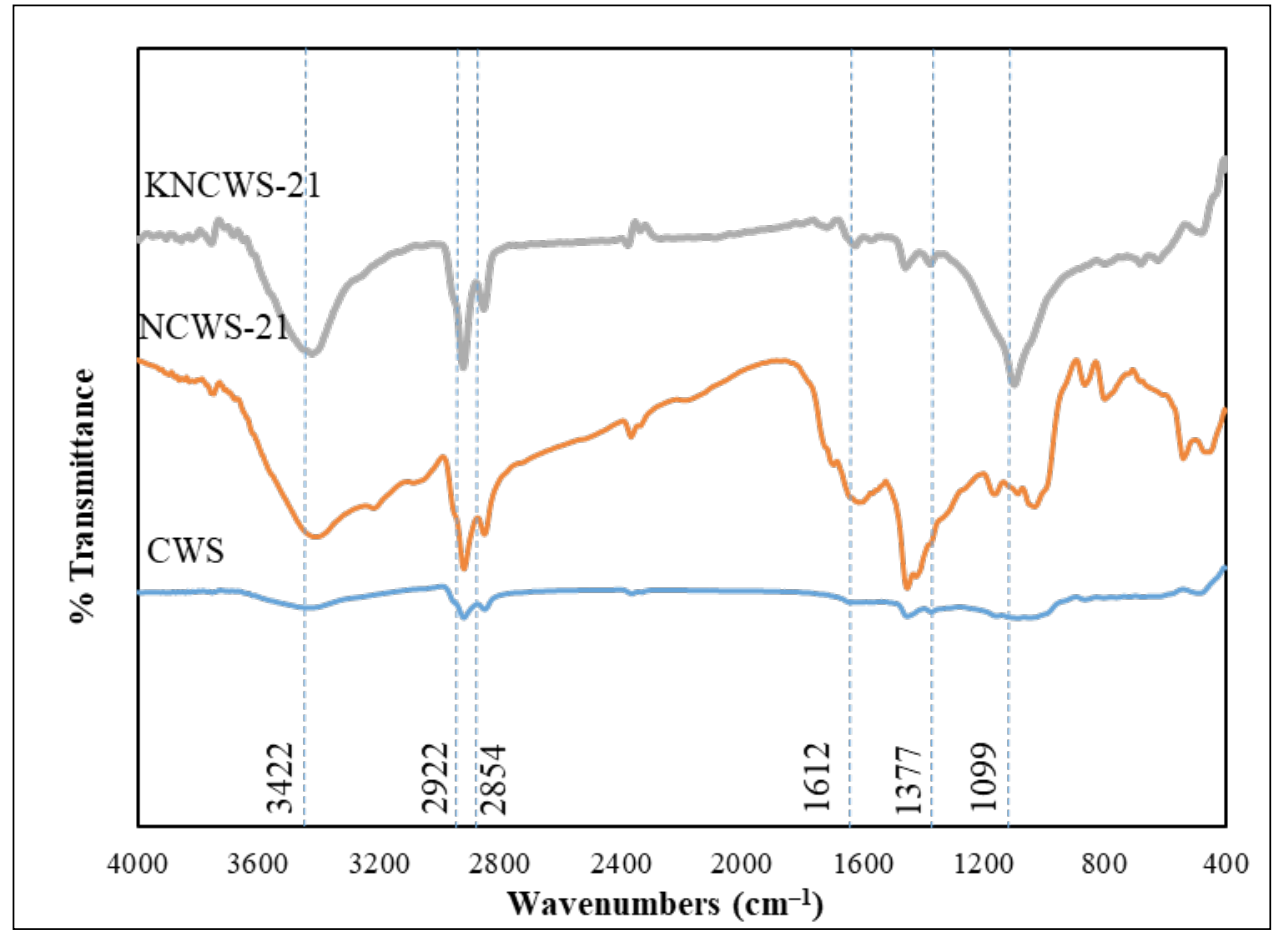

Fig. 4. FT-IR spectra of the as-synthesized KNCWs 


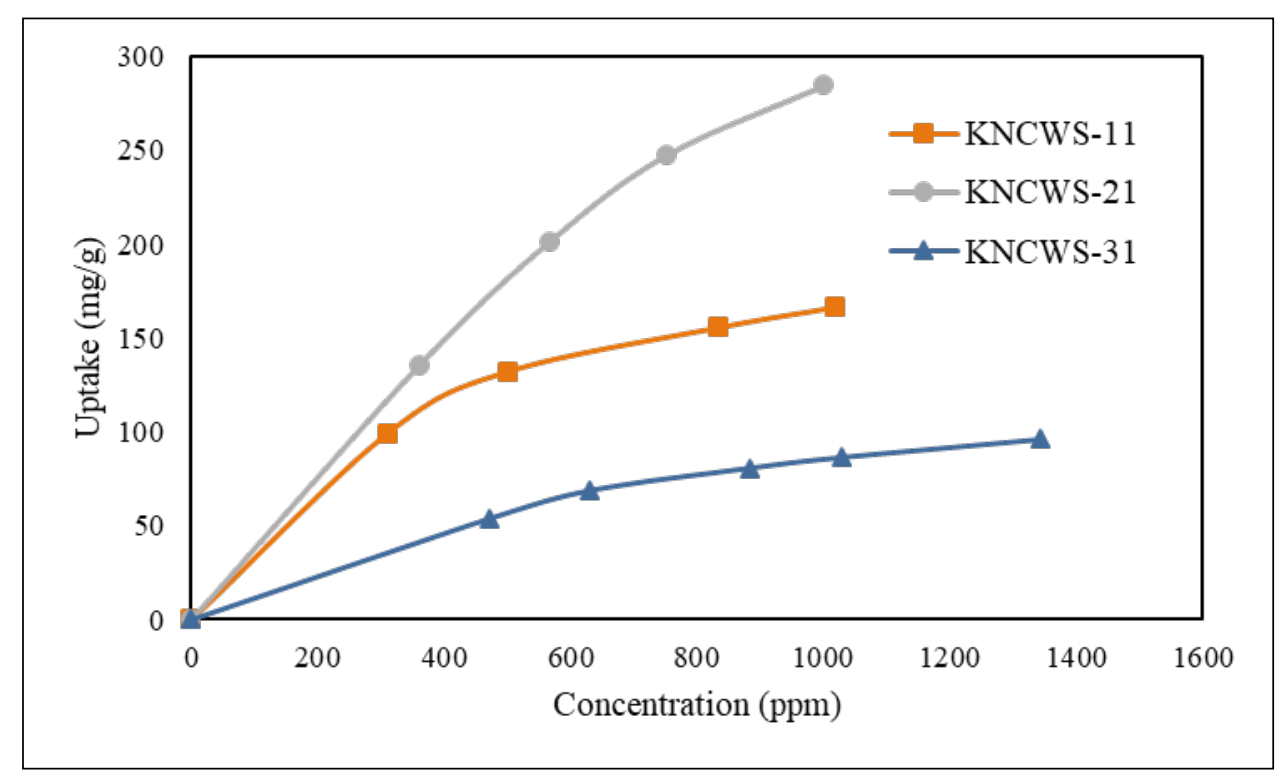

Fig. 5. Isotherm of PCE adsorption on KNCWs

nature of PCE is electron-accepting, and nitrogen groups are electron-giving and cause chemical adsorption. It should be noted that the high share of mesopores also facilitates the transfer of PCE mass and increases adsorption. According to the results of PCE adsorption, it can be acknowledged that the reason for the significant decrease in the adsorption rate of the KNCWS-31 sample is the decrease in its physical characteristics, which occurred due to the destruction of the walls between cavities or blockage of cavities with excessive nitrogen.

According to Table 2, the modulus of adsorption equilibrium data for the adsorption of PCE on doped activated nano carbons was consistent with the Langmuir, Freundlich, and Sips models [20]. However, the Sips model showed higher values of $\mathrm{R}^{2}$ than the Langmuir and Freundlich models for all samples studied. In this experiment, the b-constant of the Sips model was approximately zero, indicating that the Sips model was reduced to the Freundlich model. This means that multilayer adsorption occurs with a non-uniform distribution of heat and tensile adsorption across the surface of doped activated nano table 2.

\subsection{Kinetic adsorption}

In order to evaluate the speed of the adsorption process and to determine the process speed control stage, kinetic modeling is performed. Kinetic data were evaluated using pseudo-first-order [21] and pseudo-second-order [22] models to provide a suitable model for the kinetic behavior of the studied gravity (Fig. 6).

The adsorption kinetics and correlation coefficients of PCE on doped and non-doped activated nano carbons are shown in Table 3. Both pseudo-firstorder and pseudo-second-order equations can predict the adsorption process under experimental conditions. Given the calculated values of $\mathrm{q}_{\mathrm{e}}$ and $\mathrm{R}^{2}$, the pseudo-first-order equation better describes the adsorption of PCE. The values of $\mathrm{R}^{2}$ in the pseudofirst-order equation have higher values than the pseudo-second-order equation. The results show that the adsorption of PCE in doped activated nano carbons belongs to the pseudo-first-order equation, which indicates the reversible adsorption between PCE and the adsorbent table 3.

\section{Conclusion}

In this study, a series of nitrogen-doped activated nano carbons were synthesized using walnut shell and evaluated to adsorb perchloroethylene (PCE) vapor. In practice, the vapor adsorption capacity of PCE using nitrogen-doped activated carbon 
Table 2. The parameters of Langmuir, Freundlich, and Sips isotherms of PCE adsorption on doped activated nano carbons

\begin{tabular}{|c|c|c|c|c|}
\hline \multicolumn{2}{|c|}{ Isotherms } & \multirow[b]{2}{*}{ KNCWS-11 } & \multicolumn{2}{|c|}{ Adsorbents } \\
\hline & & & KNCWS-21 & KNCWS-31 \\
\hline \multirow[t]{2}{*}{ Langmuir } & $\mathrm{q}_{\mathrm{m}}$ & 348.3383 & 703.6625 & 168.2004 \\
\hline & $\mathrm{b}$ & 0.00065812 & 0.00069629 & 0.0010431 \\
\hline$q_{e}=q_{m} \frac{b P}{1+b P}$ & R-squared & 0.99962 & 0.99824 & 0.99847 \\
\hline \multirow[t]{2}{*}{ Freundlich } & $\mathrm{q}_{\mathrm{m}}$ & 50.3955 & 179.2698 & 23.3665 \\
\hline & $\mathrm{b}$ & 0.0042402 & 0.0020152 & 0.009701 \\
\hline \multirow[t]{2}{*}{$\mathbf{q}_{\mathrm{e}}=\mathbf{q}_{\mathrm{m}}(\mathbf{b} \mathbf{P})^{1 / \mathrm{n}}$} & $\mathrm{n}$ & 1.4203 & 1.4426 & 1.7413 \\
\hline & R-squared & 0.99839 & 0.99586 & 0.99721 \\
\hline \multirow[t]{2}{*}{ Sips } & $\mathrm{q}_{\mathrm{m}}$ & 232.0382 & 399.4676 & 100.7033 \\
\hline & $\mathrm{b}$ & 0.0013378 & 0.0018034 & 0.0022754 \\
\hline \multirow{2}{*}{$q_{e}=q_{m} \frac{(b P)^{1 / n}}{1+(b P)^{1 / n}}$} & $\mathrm{n}$ & 0.78099 & 0.64366 & 0.48246 \\
\hline & R-squared & 1.00000 & 0.99989 & 0.99979 \\
\hline
\end{tabular}

Table 3. Pseudo-first and second-order kinetics parameters of PCE adsorption on doped activated nano carbons

\begin{tabular}{|c|c|c|c|c|}
\hline \multirow{2}{*}{\multicolumn{2}{|c|}{$\begin{array}{c}\text { Isotherms } \\
\text { KNCWS-11 }\end{array}$}} & \multicolumn{3}{|c|}{ Adsorbents } \\
\hline & & \multirow{2}{*}{$\frac{\text { KNCWS-21 }}{140.582}$} & \multirow{2}{*}{$\frac{\text { KNCWS-31 }}{295.911}$} & \multirow[b]{2}{*}{84.529} \\
\hline \multirow[b]{3}{*}{$\mathbf{q}_{\mathrm{t}}=\mathbf{q}_{\mathrm{e}}\left(1-\exp \left(-K_{1} t\right)\right)$} & $\mathrm{q}_{\mathrm{e}}$ & & & \\
\hline & $\mathrm{K}_{1}$ & 0.0309 & 0.031 & 0.095 \\
\hline & R-squared & 0.990 & 0.982 & 0.981 \\
\hline pseudo-second-order & $\mathrm{q}_{\mathrm{e}}$ & 176.946 & 382.646 & 0.001 \\
\hline \multirow{2}{*}{$\mathrm{q}_{\mathrm{t}}=\frac{K_{2} q_{e}^{2} t}{1+K_{2} \mathrm{q}_{\mathrm{e}} t}$} & $\mathrm{~K}_{2}$ & 0.0001 & 7.603 & 0.001 \\
\hline & R-squared & 0.970 & 0.960 & 0.995 \\
\hline
\end{tabular}

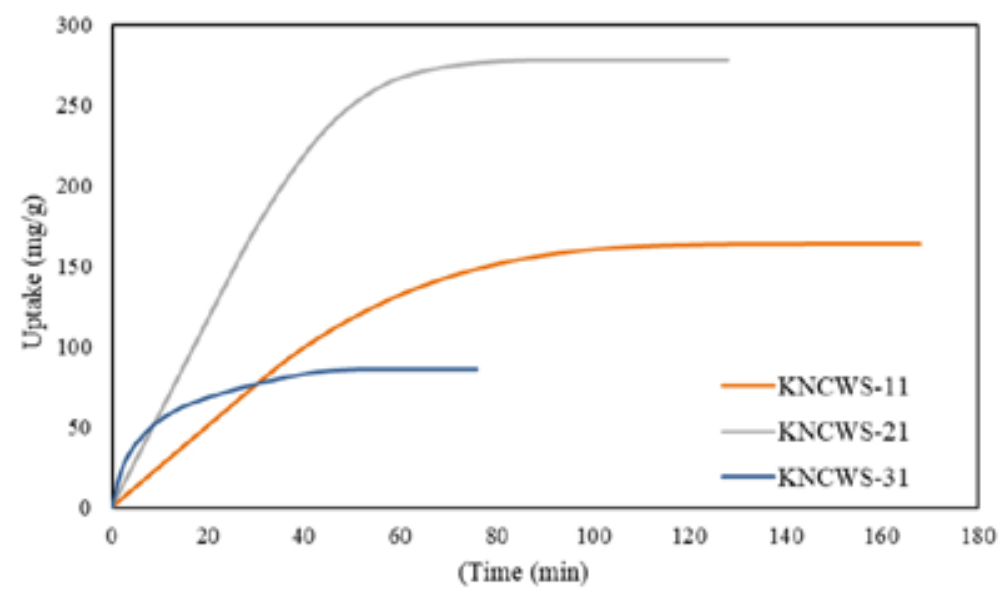

Fig. 6. Kinetics of PCE adsorption on KNCWs 
varies considerably due to their structural and surface roperties. Nitrogen plays an important role in the adsorption of PCE. Samples of KNCWS-11, KNCWS-21, and KNCWS-31 at initial concentrations of $1000 \mathrm{ppm}$ have adsorption rates of 166,285 , and $95 \mathrm{mg} \mathrm{g}^{-1}$, respectively. Therefore, the use of nitrogen-doped activated nano carbons as a green adsorbent provides a costeffective means of combating biomass waste and can partially reduce the climate change caused by PCE vapor. Contaminated PCE/ $\mathrm{N}_{2}$ at the inlet and outlet of the column was analyzed online with GCMS analyzer.

\section{Acknowledgments}

The authors wish to thank the Chemical Engineering Department, Islamic Azad University, North Tehran Branch, Tehran, Iran

\section{References}

[1] M. Słomińska, P. Konieczka, J. Namieśnik, The fate of BTEX compounds in ambient air, Crit. Rev. Environ. Sci. Technol., 44 (2014) 455-472.

[2] L. Yu, L. Wang, W. Xu, L. Chen, M. Fu, J. Wu, D. Ye, Adsorption of VOCs on reduced graphene oxide, J. Environ. Sci., 67 (2018) 171-178.

[3] S. Jafari, F. Ghorbani-Shahna, A. Bahrami, H. Kazemian, Adsorptive removal of toluene and carbon tetrachloride from gas phase using zeolitic imidazolate framework-8: Effects of synthesis method, particle size, and pretreatment of the adsorbent, Microporous Mesoporous Mater., 268 (2018) 58-68.

[4] C. Dai, Y. Zhou, H. Peng, S. Huang, P. Qin, J. Zhang, Y. Yang, L. Luo, X. Zhang, Current progress in remediation of chlorinated volatile organic compounds: A review, J. Ind. Eng. Chem., 62 (2018) 106-119.

[5] R.E. Doherty, A history of the production and use of carbon tetrachloride, tetrachloroethylene, trichloroethylene and 1,1,1-trichloroethane in the United States: Part 1--historical background; carbon tetrachloride and tetrachloroethylene,
Environ. Forensics., 1 (2000) 69-81.

[6] B. Huang, C. Lei, C. Wei, G. Zeng, Chlorinated volatile organic compounds (Cl-VOCs) in environment sources, potential human health impacts, and current remediation technologies, Environ. Int., 71 (2014) 118138.

[7] C. Barton, Tetrachloroethylene, in: P. Wexler (Ed.), Encycl. Toxicol. (Third Ed., Third Edition, Academic Press, Oxford, pp. 498-502, 2014. https://doi.org/https://doi. org/10.1016/B978-0-12-386454-3.00436-X.

[8] J.D. Tucker, K.J. Sorensen, A.M. Ruder, L.T. McKernan, C.L. Forrester, M.A. Butler, Cytogenetic analysis of an exposed-referent study: perchloroethylene-exposed dry cleaners compared to unexposed laundry workers, Environ. Heal., 10 (2011) 16.

[9] Y. Zeng, Z. Zeng, T. Ju, F. Zhang, Adsorption performance and mechanism of perchloroethylene on a novel nano composite $\beta$-FeOOH-AC, Micropor. Mesopor. Mater., 210 (2015) 60-68.

[10] Z. Rouzitalab, D.M. Maklavany, S. Jafarinejad, A. Rashidi, Lignocellulose-based adsorbents: A spotlight review of the effective parameters on carbon dioxide capture process, Chemosphere, 246 (2020). https://doi. org/10.1016/j.chemosphere.2019.125756.

[11] E. Jangodaz, E. Alaie, A.A. Safekordi, S. Tasharrofi, Adsorption of ethylbenzene from air on metal-organic frameworks MIL-101(Cr) and MIL-53(Fe) at room temperature, J. Inorg. Organomet. Polym. Mater., 28 (2018) 2090-2099.

[12] M. Thommes, K. Kaneko, A. V Neimark, J.P. Olivier, F. Rodriguez-Reinoso, J. Rouquerol, K.S.W. Sing, Physisorption of gases, with special reference to the evaluation of surface area and pore size distribution (IUPAC Technical Report), Pure Appl. Chem., 87 (2015) 1051-1069.

[13] M.S. Shafeeyan, W.M.A.W. Daud, A. Houshmand, A. Arami-Niya, Ammonia modification of activated carbon to enhance 
carbon dioxide adsorption: Effect of preoxidation, Appl. Surf. Sci., 257 (2011) 39363942.

[14] Z. Rouzitalab, D. Mohammady Maklavany, A. Rashidi, S. Jafarinejad, Synthesis of $\mathrm{N}$-doped nanoporous carbon from walnut shell for enhancing $\mathrm{CO} 2$ adsorption capacity and separation, J. Environ. Chem. Eng., 6 (2018) 6653-6663.

[15] J. Chen, J. Yang, G. Hu, X. Hu, Z. Li, S. Shen, M. Radosz, M. Fan, Enhanced CO2 capture capacity of nitrogen-doped biomass-derived porous carbons, ACS Sustain. Chem. Eng., 4 (2016) 1439-1445.

[16] A. Heidari, H. Younesi, A. Rashidi, A.A. Ghoreyshi, Evaluation of $\mathrm{CO} 2$ adsorption with eucalyptus wood based activated carbon modified by ammonia solution through heat treatment, Chem. Eng., J. 254 (2014) 503513.

[17] A. Kongnoo, P. Intharapat, P. Worathanakul, C. Phalakornkule, Diethanolamine impregnated palm shell activated carbon for $\mathrm{CO} 2$ adsorption at elevated temperatures, J. Environ. Chem. Eng., 4 (2016) 73-81.

[18] Z. Xiong, Z. Shihong, Y. Haiping, S. Tao, C. Yingquan, C. Hanping, Influence of NH3/ $\mathrm{CO} 2$ modification on the characteristic of biochar and the $\mathrm{CO} 2$ capture, Bioenergy Res., 6 (2013) 1147-1153.

[19] Z. Geng, Q. Xiao, H. Lv, B. Li, H. Wu, Y. Lu, C. Zhang, One-step synthesis of microporous carbon monoliths derived from biomass with high nitrogen doping content for highly selective CO2 capture, Sci. Rep., 6 (2016) 4-11.

[20] N.S. Nasri, U.D. Hamza, S.N. Ismail, M.M. Ahmed, R. Mohsin, Assessment of porous carbons derived from sustainable palm solid waste for carbon dioxide capture, J. Clean. Prod., 71 (2014) 148-157.

[21] N. Álvarez-Gutiérrez, M. V. Gil, F. Rubiera, C. Pevida, Kinetics of $\mathrm{CO} 2$ adsorption on cherry stone-based carbons in $\mathrm{CO} 2 / \mathrm{CH} 4$ separations, Chem. Eng. J., 307 (2017) 249-
257.

[22] Y.S. Ho, G. McKay, Pseudo-second order model for sorption processes, Process Biochem., 34 (1999) 451-465. 